\title{
HANKEL COMPLEMENTARY INTEGRAL TRANSFORMATIONS OF ARBITRARY ORDER
}

\author{
M. LINARES LINARES \\ Departamento de Informática y Sistemas, \\ Universidad de Las Palmas, Las Palmas de Gran Canaria \\ Canary Islands, Spain

\section{J.M.R. MENDEZ PÉREZ} \\ Departamento de Análisis Matemático, Facultad de Matemáticas, \\ Universidad de La Laguna, La Laguna (Tenerife) \\ Canary Islands, Spain
}

(Received November 13, 1990 and in revised form June 18, 1991)

Abstract. Four selfreciprocal integral transformations of Hankel type are defined through

$$
\left(\mathscr{H}_{i, \mu} f\right)(y)=F_{i}(y)=\int_{0}^{\infty} \alpha_{i}(x) g_{i, \mu}(x y) f(x) d x, \mathscr{H}_{i, \mu}^{1}=\mathcal{H}_{i, \mu},
$$

where $i=1,2,3,4 ; \mu \geq 0 ; \alpha_{1}(x)=x^{1+2 \mu}, g_{1, \mu}(x)=x^{-\mu} J_{\mu}(x), J_{\mu}(x)$ being the Bessel function of the first kind of order $\mu ; \alpha_{2}(x)=x^{1-2 \mu}, g_{2, \mu}(x)=(-1)^{\mu} x^{2 \mu} g_{1, \mu}(x) ; \alpha_{3}(x)=x^{-1-2 \mu}, g_{3, \mu}(x)=x^{1+2 \mu} g_{1, \mu}(x)$, and $\alpha_{4}(x)=x^{-1+2 \mu}$, $g_{4, \mu}(x)=(-1)^{\mu} x g_{1, \mu}(x)$. The simultaneous use of transformations $\mathscr{H}_{1, \mu}$ and $\mathscr{H}_{2, \mu}$ (which are denoted by $\mathscr{H}_{\mu}$ ) allows us to solve many problems of Mathematical Physics involving the differential operator $\Delta_{\mu}=D^{2}+(1+2 \mu) x^{-1} D$, whereas the pair of transformations $\mathscr{H}_{3, \mu}$ and $\mathscr{H}_{4, \mu}$ (which we express by $\mathcal{H}_{\mu}$ ) permits us to tackle those problems containing its adjoint operator $\Delta_{\mu}^{*}=D^{2}-(1+2 \mu) x^{-1} D+(1+2 \mu) x^{-2}$, no matter what the real value of $\mu$ be. These transformations are also investigated in a space of generalized functions according to the mixed Parseval equation

$$
\int_{0}^{\infty} f(x) g(x) d x=\int_{0}^{\infty}\left(\mathcal{H}_{\mu} f\right)(y)\left(\mathcal{H}_{\mu}^{\infty} g\right)(y) d y,
$$

which is now valid for all real $\mu$.

Key Words and Phrases: Complementary Hankel transformations, Parseval equation, generalized functions.

1980 Mathematics Subject Classification: Primary 46F12, 44A05

\section{INTRODUCTION.}

It was proved by A.H. Zemanian $[15,17]$ that the Hankel transformation of order $\mu \geq-1 / 2$

$$
\left(h_{\mu} f\right)(y)=\int_{0}^{\infty} \sqrt{x y} J_{\mu}(x y) f(x) d x,
$$

where $J_{\mu}(x)$ denotes the Bessel function of the first kind, is an automorphism on the space $\mathbf{H}_{\mu}$ of the infinitely differentiable complex-valued functions $\phi(x), x \in(0, \infty)$, such that

$$
\gamma_{m, k}^{\mu}(\phi)=\sup _{0<x<\infty}\left|x^{m}\left(x^{-1} D\right)^{k} x^{-\mu-1 / 2} \phi(x)\right|
$$


exists for each pair of nonnegative integers $m$ and $k$. Then, the generalized Hankel transformation $h_{\mu}^{\prime}$ is defined on the dual space $\mathbf{B}_{\mu}^{\prime}$ by means of the adjoint operator of $h_{\mu}$, that is,

$$
\left\langle h^{\prime}{ }_{\mu}, \Phi\right\rangle=\left\langle f, h_{\mu} \Phi\right\rangle, f \in H_{\mu}^{\prime}, \Phi \in H_{\mu} .
$$

We emphasize that the last expression appears as a generalization of a well-known Parseval equation for the Hankel transformation ([17,p. 127],[6]). Consequently, the generalized transformation $h^{\prime}{ }_{\mu}$ is also an automorphism on the space $\mathbf{B}_{\mu}^{\prime}$ provided that $\mu \geq-1 / 2$. Later, A.H. Zemanian [16] and E.L. Koh [4] remove the restriction $\mu \geq 1 / 2$ and assume that $\mu$ be any fixed real number. For it, a positive integer $r$ is chosen such that $\mu+r \geq-1 / 2$ and then the Hankel transformation of arbitrary order $\mu$ is given by

$$
\left(h_{\mu, r} \phi\right)(y)=(-1)^{r} y^{-r}\left[h_{\mu+r}\left(N_{\mu+r-1} \ldots \ldots N_{\mu+1} N_{\mu} \phi\right](y)\right. \text {, }
$$

where the operator

$$
N_{\mu}=x^{\mu+1 / 2} D x^{-\mu-1 / 2}
$$

generates an isomorphism from the space $\mathbf{B}_{\mu}$ onto $\mathbf{B}_{\mu+1}$ and the mapping $\phi(x) \rightarrow x^{r} \phi(x)$ is an isomorphism between the spaces $\mathbf{B}_{\mu}$ and $\mathbf{B}_{\mu+r}$, as well. The main idea of this method consists in leading any member $\phi(x) \in \mathbf{B}_{\mu}$, by means of the applications of the operator $N_{\mu}, N_{\mu+1}, \ldots \ldots$ and $N_{\mu+r-1}$ one after another, to the space $\mathbf{B}_{\mu+r}$ where the inversion formula of the Hankel transform has already a sense since $\mu+r \geq 1 / 2$. Recently we have employed this procedure to extend to an arbitrary order $\mu$ the following variant of the Hankel transformation ([8]):

$$
\left(\mathcal{H}_{1, \mu} f\right)(y)=F_{1}(y)=\int_{0}^{\infty} x^{1+2 \mu} g_{1, \mu}(x y) f(x) d x
$$

where $g_{1, \mu}(x)=x^{-\mu} J_{\mu}(x)$. The transform (1.2) is called in the available literature the Schwartz's Hankel transformation.

The principal aim of this paper is to extend the last transformation (1.2) to any real value of $\mu$, through a technique quite different from that was used in the previous works [4], [8] and [16].

To attain a more symmetrical expression for our results, from now on we assume that $\mu \geq 0$. $L(I)$ denotes the space of all functions $f(x)$ that are Lebesgue integrable on the real positive axis $I=(0, \infty) . D(I)$ stands for the space of infinitely differentiable functions whose supports are contained in $I$ and its dual $D^{\prime}(I)$ is the space of Schwartz distributions [11]. Finally, $E(I)$ represents the space of all infinitely differentiable functions on $I$ and its dual $E^{\prime}(I)$ is the space of distributions with compact supports [17,p.36].

\section{Classical Results on Schwartz's Hankel Transformations.}

In relation with the transformation (1.2) we establish

Theorem 2.1. Let $\mu \geq 0$. If $y^{\mu+1 / 2} f(y) \in L(I)$, then

$$
\int_{0}^{\infty} y^{1+2 \mu} g_{1, \mu}(x y) F_{1}(y) d y=\frac{1}{2}[f(x+0)+f(x-0)]
$$

in a neighborhood of every point $y=x$ where $f(y)$ is of bounded variation.

Proof. The assertion follows from the relation

$$
\left(\mathcal{H}_{1, \mu} f\right)(y)=y^{-\mu-1 / 2}\left\{h_{\mu}\left(x^{\mu+1 / 2} f(x)\right)\right\}(y)
$$

between (1.1) and (1.2) and from the inversion theorem for the Hankel transformation [13,p.240].

Other conditions under which Theorem 2.1 holds were proposed by A.L. Schwartz [12].

Note that the function $g_{1, \mu}(x y)$ satisfies the equation

$$
\left(\Delta_{1, \mu, x}+y^{2}\right) g_{1, \mu}(x y)=0
$$

where 


$$
\Delta_{1, \mu, x}=\Delta_{1, \mu}=D^{2}+(1+2 \mu) x^{-1} D, D=\frac{d}{d x}
$$

If $f(x)$ and $G_{1}(y)$ are functions defined on $I$ such that $x^{\mu+12} f(x) \in L(I)$ and $y^{\mu+12} G_{1}(y) \in L(I)$ and if $F_{1}(y)=\left(\mathscr{H}_{1, \mu} f\right)(y)$ and $g(x)=\left(\mathcal{H}_{1, \mu}^{1} G_{1}\right)(x)=\left(\mathcal{H}_{1, \mu} G_{1}\right)(x)$, where $\mu \geq 0$, then we easily obtain the Parseval equation

$$
\int_{0}^{\infty} x^{1+2 u} f(x) g(x) d x=\int_{\infty}^{\infty} y^{1+2 u} F_{1}(y) G_{1}(y) d y
$$

In previous years, Schwartz's Hankel transformation has been investigated in certain spaces of distributions, amongst other authors, by L.S. Dube and J.N. Pandy [2], W.Y. Lee [5], A. Schuitman [10] and G. Altenburg [1].

Next, we introduce the transformation

$$
\left(\mathcal{H}_{2, \mu} g\right)(y)=G_{2}(y)=\int_{0}^{\infty} x^{1-2 \mu} g_{2, \mu}(x y) g(x) d x
$$

where $g_{2, \mu}(x)=(-1)^{\mu} x^{2 \mu} g_{1, \mu}(x)$ fulfills the equation

$$
\left(\Delta_{2, \mu, x}+y^{2}\right) g_{2, \mu}(x y)=0,
$$

$\Delta_{\mathbf{2}, \mu}$ denoting the differential operator

$$
\Delta_{2, \mu, x}=\Delta_{2, \mu}=D^{2}+(1-2 \mu) x^{-1} D
$$

Observe that the multiplication by $x^{2 \mu}$ only implies the change of sign for the parameter $\mu$ in (2.1) to convert it into equation (2.4). On the other hand, transformations (1.2) and (2.3) are closely related, as it is made evident by the expression

$$
\left(\mathcal{H}_{2, \mu} g\right)(y)=(-1)^{\mu} y^{2 \mu}\left[\mathscr{H}_{1, \mu}\left(x^{-2 \mu} g(x)\right)\right](y)
$$

Then, from Theorem 2.1 and (2.5) it is immediately inferred the classical inversion formula for the transformation $\mathcal{H}_{2, \mu}$ :

Theorem 2.2. Let $\mu \geq 0$. If $y^{-\mu+1 / 2} g(y) \in L(I)$ and $G_{2}(y)$ is defined as in (2.3), then we have

$$
\int_{0}^{\infty} y^{1-2 \mu} g_{2, \mu}(x y) G_{2}(y) d y=\frac{1}{2}[g(x+0)+g(x-0)]
$$

in a neighborhood of every point $y=x$ where $g(y)$ is of bounded variation. For a pair of functions $f(x)$ and $G_{2}(y)$ such that $x^{-\mu+1 / 2} f(x)$ and $y^{-\mu+1 / 2} G_{2}(y)$ belong to $L(I)$, the following Parseval equation

$$
\int_{0}^{\infty} x^{1-2 u} f(x) g(x) d x=\int_{0}^{\infty} y^{1-2 \mu} F_{2}(y) G_{2}(y) d y
$$

is valid, where $F_{2}(y)=\left(\mathcal{H}_{2, \mu} f\right)(y), g(x)=\left(\mathcal{H}_{2, \mu}^{1} G_{2}\right)(x)=\left(\mathcal{H}_{2, \mu} G_{2}\right)(x)$ and $\mu \geq 0$.

According to the relation (2.5) the transforms $\mathscr{H}_{1, \mu}$ and $\mathscr{H}_{2, \mu}$ coincide seemingly when and only when $\boldsymbol{\mu}=\mathbf{0}$. However, it is worth to remark that there are other values of $\boldsymbol{\mu}$ which make equal the transformations $\mathscr{H}_{1, \mu}$ and $\mathscr{H}_{2, \mu}$. Indeed, from the relation [13,p.16]

$$
g_{1,-n}(x)=g_{2, n}, n=0,1,2,3, \ldots \ldots,
$$

we deduce quickly that

$$
\left(\mathcal{H}_{1,-n} f\right)(y)=\left(\mathcal{H}_{2, n} f\right)(y),
$$

in other words, the transformation $\mathcal{H}_{2, n}$ of positive integer order might be used to replace the transformation $\mathscr{H}_{1, \mu}$ of negative integer index. This fact and above considerations suggest to adopt the notation

$$
\Delta_{\mu}=\Delta_{\mu, x}=D^{2}+(1+2 \mu) x^{-1} D=x^{-1-2 \mu} D x^{1+2 \mu} D,-\infty<\mu<\infty,
$$


and

$$
b_{\mu}(x)= \begin{cases}g_{1, \mu}(x), & \text { if } \mu \geq 0 \\ g_{2,-\mu}(x), & \text { if } \mu<0\end{cases}
$$

Now we assemble (1.2) and (2.3) in the unique expression

$$
\left(\mathcal{H}_{\mu} f\right)(y)=F(y)=\int_{0}^{\infty} x^{1+2 \mu} b_{\mu}(x y) f(x) d x
$$

Therefore Theorems 2.1 and 2.2 can be enunciated together:

Theorem 2.3. Let $\mu$ be an arbitrary real number. If the function $f(y)$ is such that $x^{\mu+1 / 2} f(x) \in L(I)$, if $f(y)$ is of bounded variation in a neighborhood of $y=x \in I$ and if $F(y)$ is given by (2.8), then

$$
\int_{0}^{\infty} y^{1+2 \mu} b_{\mu}(x y) F(y) d y=\frac{1}{2}[f(x+0)+f(x-0)]
$$

In [7] we studied this other variant of the Hankel transformation

$$
\left(\mathcal{H}_{3, \mu} f\right)(y)=F_{3}(y)=\int_{0}^{\infty} x^{-1-2 \mu} g_{3, \mu}(x y) f(x) d x,
$$

where $g_{3, \mu}(x)=x^{2 \mu+1} g_{1, \mu}$ turns out to be a solution of the equation

$$
\left(\Delta_{3, \mu, x}+y^{2}\right) g_{3, \mu}(x y)=0
$$

$\Delta_{3, \mu}$ being the differential operator

$$
\Delta_{3, \mu, x}=\Delta_{3, \mu}=D^{2}-(1+2 \mu) x^{-1} D+(1+2 \mu) x^{-2}
$$

The following inversion formula was derived in [7]:

Theorem 2.4. Let $\mu \geq 0$. If $f(y)$ is a function defined on $I$ such that $y^{-\mu-1 / 2} f(y) \in L(I)$, then

$$
\int_{0}^{\infty} y^{-1-2 \mu} g_{3, \mu}(x y) F_{3}(y) d y=\frac{1}{2}[f(x+0)+f(x-0)]
$$

in a neighborhood of every point $y=x$ where $f(y)$ is of bounded variation.

Moreover, if $f(x)$ and $G_{3}(y)$ are two functions defined over the positive real axis such that $x^{-\mu-1 / 2} f(x)$ and $y^{-\mu-1 / 2} G_{3}(y)$ belong to $L(I)$, we obtain the Parseval equation

$$
\int_{0}^{\infty} x^{-1-2 u} f(x) g(x) d x=\int_{0}^{\infty} y^{-1-2 \mu} F_{3}(y) G_{3}(y) d y
$$

where $F_{3}(y)=\left(\mathscr{H}_{3, \mu} f\right)(y), g(x)=\left(\mathscr{H}_{3, \mu}^{1} G_{3}\right)(x)=\left(\mathcal{H}_{3, \mu} G_{3}\right)(x)$ and $\mu \geq 0$.

Finally, we introduce a fourth integral transformation by means of

$$
\left(\mathcal{H}_{4, \mu} g\right)(y)=G_{4}(y)=\int_{0}^{\infty} x^{-1+2 \mu} g_{4, \mu}(x y) g(x) d x,
$$

in whose kernel appears the function $g_{4, \mu}(x)=(-1)^{\mu} x g_{1, \mu}(x)=(-1)^{\mu} x^{-2 \mu} g_{3, \mu}(x)$,

which is a solution of the equation

$$
\left(\Delta_{4, \mu, x}+y^{2}\right) g_{4, \mu}(x y)=0 .
$$

Here $\Delta_{4, \mu}$ symbolizes the differential operator

$$
\Delta_{4, \mu, x}=\Delta_{4, \mu}=D^{2}-(1-2 \mu) x^{-1} D+(1-2 \mu) x^{-2}
$$

From the relation

$$
\left(\mathscr{H}_{4, \mu} g\right)(y)=(-1)^{\mu} y\left[\mathscr{H}_{1, \mu}\left(x^{-1} g(x)\right)\right](y)
$$

between transformations (1.2) and (2.12), by virtue of Theorem 2.1, we can easily state the inversion formula for the new transform: 
Theorem 2.5. Let $\mu \geq 0$. If $g(y)$ is a function on $I$ such that $y^{\mu-1 / 2} g(y) \in L(I)$, if $g(y)$ is of bounded variation in a neighborhood of the point $y=x \in I$ and $G_{4}(y)$ is given by (2.12), then

$$
\int_{0}^{\infty} y^{-1+2 \mu} g_{4, \mu}(x y) G_{4}(y) d y=\frac{1}{2}[g(x+0)+g(x-0)]
$$

Another result, which we shall need, is the corresponding Parseval equation that now takes the form

$$
\int_{0}^{\infty} x^{-1+2 u} f(x) g(x) d x=\int_{0}^{\infty} y^{-1+2 u} F_{4}(y) G_{4}(y) d y,
$$

where $F_{4}(y)=\left(\mathscr{H}_{4, \mu} f\right)(y), g(x)=\left(\mathscr{F}_{4, \mu}^{-1} G_{4}\right)(x)=\left(\mathcal{H}_{4, \mu} G_{4}\right)(x)$ and $\mu \geq 0, f(x)$ and $G_{4}(y)$ being a pair of functions such that $x^{\mu-1 / 2} f(x)$ and $y^{\mu-1 / 2} G_{4}(y)$ belong to $L(I)$.

By perusing Theorem 2.4 and 2.5, Parseval relations (2.11) and (2.15) and differential operators $\Delta_{3, \mu}$ and $\Delta_{4, \mu}$ the unique apparent difference lies in the change of the sign on the parameter $\mu$. In the same way, it is convenient to point out that the multiplication of $g_{3, \mu}(x)$ by $(-1)^{\mu} x^{-2 \mu}$ to get the function $g_{4, \mu}(x)$ exactly implies the said change of sign. Furthermore, it is easily seen that

$$
\left(\mathscr{H}_{3, \rightarrow n} g\right)(y)=\left(\mathscr{H}_{4, n} g\right)(y) \text {, }
$$

since $g_{3,-n}(x)=g_{4, n}(x), n=0,1,2,3, \ldots \ldots$. Now, it is wholly justified to adopt the following notation

$$
\Delta_{\mu}^{*}=\Delta_{4, \mu}=D^{2}-(1+2 \mu) x^{-1} D+(1+2 \mu) x^{-2}=D x^{1+2 \mu} D x^{-1-2 \mu},-\infty<\mu<\infty
$$

and

$$
b_{\mu}^{*}(x)= \begin{cases}g_{3, \mu}(x), & \text { if } \mu \geq 0 \\ g_{4,-\mu}(x), & \text { if } \mu \leq 0\end{cases}
$$

Thus, the transformations (2.9) and (2.12) can be rewritten in the unique expression

$$
\left(\mathcal{H}_{\mu}^{\prime} g\right)(y)=G^{*}(y)=\int_{0}^{\infty} x^{-1-2 \mu} b_{\mu}^{*}(x y) g(x) d x
$$

Next, we summarize Theorems 2.4 and 2.5 :

Theorem 2.6. If $g(y)$ is a function defined on $I$ such that $y^{-\mu-1 / 2} g(y) \in L(I)$, if $g(y)$ is of bounded variation in a neighborhood of a point $y=x \in I$ and if $G^{*}(y)$ is given by (2.17), then

$$
\int_{0}^{\infty} y^{-1-2 \mu} b_{\mu}^{*}(x y) G^{*}(y) d y=\frac{1}{2}[g(x+0)+g(x-0)]
$$

for any real value of $\mu$.

A routine application of Fubini theorem allows us to establish another Parseval equations involving a pair of these four transformations $\mathcal{H}_{i, \mu}(i=1,2,3,4)$.

Theorem 2.7. Let $\mu \geq 0$.

(a) If $x^{\mu+1 / 2} f(x) \in L(I)$ and $y^{-\mu-1 / 2} G_{3}(y) \in L(I)$, then

$$
\int_{0}^{\infty} f(x) g(x) d x=\int_{0}^{\infty} F_{1}(y) G_{1}(y) d x,
$$

where $F_{1}(y)=\left(\mathcal{H}_{1, \mu} f\right)(y)$ and $G_{3}(y)=\left(\mathcal{H}_{3, \mu} g\right)(y)$.

(b) The pair of transformations $\mathscr{H}_{2, \mu}$ and $\mathscr{H}_{4, \mu}$ verifies also the mixed Parseval equation

$$
\int_{0}^{\infty} f(x) g(x) d x=\int_{0}^{\infty} F_{2}(y) G_{4}(y) d y,
$$

where now $F_{2}(y)=\left(\mathcal{H}_{2, \mu} f\right)(y)$ and $G_{4}(y)=\left(\mathcal{H}_{4, \mu} g\right)(y)$, provided that $x^{-\mu+1 / 2} f(x) \in L(I)$ and $y^{\mu-1 / 2} G_{4}(y) \in L(I)$.

Remark 1. Note that $\Delta_{3, \mu}$ and $\Delta_{4, \mu}$ are formally the adjoints operators of $\Delta_{1, \mu}$ and $\Delta_{2, \mu}$ respectively. 
By this reason, $\mathcal{H}_{3, \mu}$ will be called the adjoint or complementary transform of $\mathcal{H}_{1, \mu}$ and we shall refer to $\mathcal{H}_{4, \mu}$ as the adjoint or complementary transform of $\mathcal{H}_{2, \mu}$.

Remark 2. The Parseval equality (2.18) involves the transformation $\mathcal{H}_{1, \mu}$ and its complementary $\mathscr{H}_{3, \mu}$, whereas the Parseval formula (2.19) relates both transforms $\mathcal{H}_{2, \mu}$ and $\mathscr{H}_{4, \mu}$. Observe that, in comparison with (2.2), (2.6), (2.11) and (2.15), expressions (2.18) and (2.19) do not contain any weight function.

Remark 3. According to the notation we have just adopted, Theorem 2.7 admits this statement more concisely.

Theorem 2.8. Let $\mu$ be any fixed real number. If $x^{\mu+12} f(x)$ and $y^{-\mu-1 / 2} G^{*}(y)$ belongs to $L(I)$ and if we put $F(y)=\left(\mathcal{H}_{\mu} f\right)(y)$ and $g(x)=\left(\mathcal{H}_{\mu}^{-1} G^{*}\right)(x)=\left(\mathcal{H}_{\mu}^{*} G^{*}\right)(x)$, then

$$
\int_{0}^{\infty} f(x) g(x) d x=\int_{0}^{\infty} F(y) G^{*}(y) d y,
$$

that is,

$$
\int_{0}^{\infty} f(x) g(x) d x=\int_{0}^{\infty}\left(\mathcal{H}_{\mu} f\right)(y)\left(\mathcal{H}_{\mu} g\right)(y) d y
$$

Later on, the Parseval equality (2.20) will facilitate the extension of the transformation $\mathcal{H}_{\mu}$ and its complementary $\mathscr{H}_{\mu}^{\prime}$ to certain spaces of generalized functions [9].

\section{The Generalized Schwartz's Hankel Transformations of Arbitrary Order}

Let $\mu$ be any fixed real number and $i$ an integer, $1 \leq i \leq 4$. The space $H_{i, \mu}$ consists of all infinitely differentiable complex-valued functions $\phi(x)$ defined on $I$ for which

$$
\rho_{m, k}^{\mu, i}(\phi)=\sup _{x \in I}\left|x^{m}\left(x^{-1} D\right)^{k} w_{i}(x) \phi(x)\right|
$$

exist for each pair of nonnegative integers $m$ and $k$, where $w_{1}(x)=1, w_{2}(x)=x^{-2 \mu}, w_{3}(x)=x^{-1-2 \mu}$ and $w_{4}(x)=x^{-1}$. With the topology generated by the collections of seminorms $\left\{\rho_{m, k}^{\mu, i}\right\}, H_{i, \mu}$ are Fréchet spaces.

We denote $H_{1, \mu}=H_{1}$ and $H_{4, \mu}=H_{4}$, since the definition of these spaces is independent of the particular choice of the parameter $\mu$.

Theorem 3.1. A function $\phi(x)$ defined on $I$ is a member of $H_{i, \mu}$ if and only if, (a) $\phi(x)$ is infinitely differentiable on $I,(b) \phi(x)$ has the form

$$
\phi(x)=w_{i}^{-1}(x)\left[a_{0}+a_{1} x^{2}+a_{2} x^{4}+\ldots \ldots+a_{k} x^{2 k}+0\left(x^{2 k}\right)\right]
$$

in some vicinity of the origin, and (c) $D^{k} \phi(x)$ is of rapid descent when $x \rightarrow \infty$ for each $k=0,1,2,3, \ldots \ldots$.

$H_{i, \mu}^{\prime}$ symbolizes the dual space of $H_{i, \mu}$ and its members are generalized functions of slow growth. We assign to $H^{\prime}{ }_{i, \mu}$ the weak topology generated by the multinorm $\left\{\zeta_{i, \phi}\right\}$ defined through

$$
\zeta_{i, \phi}(f)=|\langle f, \phi\rangle|, f \in H^{\prime}{ }_{i, \mu}, \phi \in H_{i, \mu} .
$$

Thus, $H^{\prime}{ }_{i, \mu}$ is also sequentially complete.

Now we introduce the new spaces

$$
H_{\mu}=\left\{\begin{aligned}
H_{1}, & \text { if } \mu \geq 0 \\
H_{2,-\mu}, & \text { if } \mu \leq 0
\end{aligned}\right.
$$

and

$$
H_{\mu}^{*}=\left\{\begin{aligned}
H_{3, \mu}, & \text { if } \mu \geq 0 \\
H_{4}, & \text { if } \mu \leq 0
\end{aligned}\right.
$$

$H^{\prime}{ }_{\mu}$ and $H_{\mu}^{*}$, denote the dual spaces of $H_{\mu}$ and $H_{\mu}^{*}$, respectively. Some properties to these spaces are listed 
below.

(i) $D(I) \subset H_{i, \mu}$ and the restriction of every $f \in H_{i, \mu}^{\prime}$ to $D(I)$ is a member of $D^{\prime}(I)$. Moreover, $H_{i, \mu}$ is a dense subspace of $E(I)$. Therefore, $E^{\prime}(I)$ is a subspace of $H_{i, \mu}^{\prime}$. Consequently, $D(I) \subset H_{\mu} \subset E(I)$ and $D(I) \subset H_{\mu}^{*} \subset E(I)$.

(ii) The operations $\phi \rightarrow \Delta_{\mu} \phi$ and $\psi \rightarrow \Delta_{\mu}^{*} \psi$, where $\Delta_{\mu}$ and $\Delta_{\mu}^{*}$ represent the operators given by (2.7) and (2.16), are continuous linear mappings from the testing-function spaces $H_{\mu}$ and $H_{\mu}^{*}$ into themselves, respectively. Indeed, the inequality

$$
\rho_{m, k}^{\mu, i}\left(\Delta_{i, \mu} \phi\right) \leq|2 k+2 \mu+2| \rho_{m, k+1}^{\mu, i}(\phi)+\rho_{m+2, k+2}^{\mu, i}(\phi), i=1,2,3,4,
$$

is satisfied for every $\phi \in H_{i, \mu}$, by taking into account that $\Delta_{i, \mu}=w_{i}^{-1}(x)\left[x^{2}\left(x^{-1} D\right)^{2}+(2+2 \mu)\left(x^{-1} D\right)\right] w_{i}(x)$. Hence, the generalized differential operator $\Delta_{\mu}$ defined on the distributional space $H_{\mu}^{*}$, as the adjoint operator of $\Delta_{\omega}^{*}$ that is to say,

$$
\left\langle\Delta_{\mu} f, \psi\right\rangle=\left\langle f, \Delta_{\mu}^{*} \psi\right\rangle, f \in H_{\mu}^{*}, \psi \in H_{\mu}^{*},
$$

is also a continuous linear mapping of $H_{\mu}^{* \prime}$ into itself. Conversely, the generalized differential operator $\Delta_{\mu}^{*}$ will be defined on $H^{\prime}{ }_{\mu}$ by means of

$$
\left\langle\Delta_{\psi}^{*} f, \psi\right\rangle=\left\langle f, \Delta_{\mu} \psi\right\rangle, f \in H^{\prime}{ }_{\mu}, \psi \in H_{\mu}
$$

and produces a continuous linear mapping of the space ${H^{\prime}}_{\mu}$ into itself.

(iii) Assume that $\mu$ is any real number. Then, $H_{\mu}$ may be identified with a subspace of $H_{\mu}^{*}$, that is, $H_{\mu} \subset H_{\mu}^{* \prime}$, the topology of $H_{\mu}$ being stronger than that induced on it by $H_{\mu}^{* \prime}$. Indeed, any $f \in H_{1}$ generates a regular distribution $f$ in the space $H_{3, \mu}^{\prime}$ by

$$
\langle f, \phi\rangle=\int_{I} f(x) \phi(x) d x, \phi \in H_{3, \mu}
$$

provided that $\mu \geq 0$. The linearity of (3.3) being obvious, the continuity is inferred from

$$
|\langle f, \phi\rangle| \leq \rho_{0,0}^{\mu, 3}(\phi) \int_{I} x^{1+2 \mu}|f(x)| d x
$$

Notice that last integral exists since $f(x)=0(1)$ when $x \rightarrow 0_{+}$and $f(x)$ is of rapid descent at infinity, by virtue of Theorem 3.1. To see the second part of the assertion observe that .

$$
\zeta_{3, \phi}(f) \leq \rho_{0,0}^{\mu, 1}(f) \int_{0}^{\infty}|\phi(x)| d x
$$

On the other hand, two members $f$ and $g$ of $H_{1}$ giving rise to the same regular distribution in $H_{3, \mu}^{\prime}$ must be identical. These considerations justify the inclusion $H_{1} \subset H_{3, \mu}^{\prime}$. Analogously, $H_{2, \mu}$ may be identified one-to-one with a subspace of $\mathrm{H}_{4}^{\prime}$, in other words, $\mathrm{H}_{2, \mu} \mathrm{CH}_{4}{ }_{4}$ whenever $\mu \leq 0$. We can now conclude, in view of notation (3.1) and (3.2), that $H_{\mu} \subset H_{\mu}^{* \prime}$. Finally, we can proceed in a similar way to get the inclusions $H_{3, \mu} \subset H^{\prime}{ }_{1}$ and $H_{4} \subset H^{\prime}{ }_{2, \mu}$ for $\mu \geq 0$ and $\mu \leq 0$, respectively, from which one deduces that $H_{\mu}^{*} \subset H_{\mu}^{\prime}$.

\section{Theorem 3.2.}

(a) The Hankel transformation $\mathcal{H}_{\mu}$, defined by (2.8), is an automorphism on the space $H_{\mu}$, no matter what the real value of $\mu$ be.

(b) The complementary Hankel transformation $\mathcal{H}_{\mu}^{\prime}$ as given by (2.17), is as well an automorphism on $H_{\mu}^{*}$, whatever be the real number $\mu$.

Proof.

(a) In [1,Th.5] it was proven thai $\mathscr{H}_{1, \mu}$ is an automorphism on $H_{1}$. To study the transformation $\mathscr{H}_{2, \mu}$ 
firstly, we note that the operation $\phi \rightarrow x^{2 \mu} \phi$ is an isomorphism from $H_{1}$ into $H_{2, \mu}$ and then we take into account the relation (2.5) between transformations $\mathcal{H}_{1, \mu}$ and $\mathcal{H}_{2, \mu}$.

(b) Since the operation $\psi \rightarrow x^{1+2 \mu} \psi$ is an isomorphism from $H_{1}$ into $H_{3, \mu}$, the expression

$$
\left(\mathcal{H}_{3, \mu} \phi\right)(y)=y^{1+2 \mu}\left[\mathcal{H}_{1, \mu}\left(x^{-1-2 \mu} \phi\right)\right](y), \phi \in H_{3, \mu},
$$

which connects the transforms (2.9) and (1.2), implies that $\mathcal{H}_{3, \mu}$ is an automorphism on $H_{3, \mu}$. Finally, in view that the mapping $\phi \rightarrow x \phi$ defines an isomorphism from $H_{1}$ into $H_{4}$ and (2.14), we deduce that $\mathcal{H}_{4, \mu}$ is an automorphism on the space $H_{4}$ as well. According to convention (2.17), we can now conclude the validity of statement (b).

Let $\mu$ be any fixed real number. We define the generalized Schwartz's Hankel transformation $\mathcal{H}_{\mu}$ of arbitrary order in the distributional space $H_{\mu}^{*}$, as the adjoint operator of $\mathcal{H}_{\mu}^{*}$ acting on $H_{\mu}^{*}$, namely,

$$
\left\langle\mathcal{H}_{\mu} f, \Phi\right\rangle=\left\langle f, \mathscr{H}_{\mu} \Phi\right\rangle
$$

for every $f \in H_{\mu}^{* \prime}$ and $\Phi \in H_{\mu}^{*}$. If we put $\phi=\mathscr{H}_{\mu} \Phi$, the application of part (b) in Theorem 3.2 allows us to rewrite (3.4) as follows

$$
\left\langle\mathcal{H}_{\mu} f, \mathcal{H}_{\mu}^{\prime} \phi\right\rangle=\langle f, \phi\rangle, f \in H_{\mu}^{*}, \phi \in H_{\mu}^{*},
$$

This expression can be understood as an extension of the Parseval equation (2.20) to distributions.

Theorem 3.3. The generalized Schwartz's Hankel transformation $\mathcal{H}_{\mu}$, given by (3.4) or (3.5), is an automorphism on $H_{\mu}^{*}$, whatever be the real value of the parameter $\mu$.

\section{Proof:}

It is a consequence of part (b) in Theorem 3.2 and [17,Th. 1.10-2].

Remark 4. Observe that the generalized transformation $\mathcal{H}_{\mu}$ is defined on the space $H_{\mu}^{*}$ as the adjoint operator of the complementary transform, and not over the space $H^{\prime}{ }_{\mu}$ by means of the adjoint operator of itself, as usual in the available literature. This is suggested and justified, at once, by the inclusion $H_{\mu} \subset H_{\mu}^{* \prime}$. Indeed, because of this inclusion if $\mathcal{H}_{\mu}$ acts on $H_{\mu}$ the most natural would be to define $\mathcal{H}_{\mu}$ on $H_{\mu}^{* \prime}$ instead of doing it on the space $H^{\prime}{ }_{\mu}$.

On the other hand, when $\mathscr{H}_{\mu}$ is defined as usual, that is,

$$
\left\langle\mathcal{H}_{\mu} f, \Phi\right\rangle=\left\langle f, \mathcal{H}_{\mu} \Phi\right\rangle, f \in H^{\prime}{ }_{\mu}, \Phi \in H_{\mu},
$$

A. Schuitman [10] found the relation

$$
\mathscr{H}_{\mu} f=y^{2 \mu+1} \mathcal{H}_{\mu}\left(x^{-2 \mu-1} f\right), f \in H_{\mu}
$$

between the classical and generalized transformations, which means that the conventional transformation $\mathscr{H}_{\mu}$ is not a particular case of generalized one.

On the contrary, if definitions (3.4) or (3.5) are adopted, $f \in H_{\mu}$ implies that $\mathcal{H}_{\mu} f \in H_{\mu}$ in view of part (a) in Theorem 3.2. Therefore, $\mathcal{H}_{\mu} f$ generates a regular member in $H_{\mu}^{* \prime}$, through (3.3),

$$
\left\langle\mathcal{H}_{\mu} f, \Phi\right\rangle=\int_{I}\left(\mathcal{H}_{\mu} f\right)(y) \Phi(y) d y, \Phi \in H_{\mu}^{*}
$$

By virtue of Parseval relation (2.20), the right-hand side of (3.7) takes the form

$$
\int_{I} f(x)\left(\mathscr{H}_{\mu}^{*} \Phi\right)(x) d x=\left\langle f, \mathscr{H}_{\mu}^{*} \Phi\right\rangle=\left\langle\mathcal{H}_{\mu} f, \Phi\right\rangle,
$$

by definition (3.4). We have arrived at

$$
\left\langle\mathcal{H}_{\mu} f, \Phi\right\rangle=\left\langle\mathcal{H}_{\mu} f, \Phi\right\rangle,
$$

for all $\Phi \in H_{\mu}^{*}$. In other words, the classical transformation $\mathcal{H}_{\mu}$ coincides now with the generalized one $\mathcal{H}_{\mu}$, when this acts on the testing-function space $H_{\mu}^{*}$. This proves that the definition (3.4) is more appropriate than (3.6). 
Remark 5. Note that the definitions of the generalized operators introduces in (ii) are consistent with the rules of distributional calculus, in particular, with the definitions of multiplication by an infinitely differentiable function and differentiation of distribution. This does not occur in [1], [5] and [10].

For every real $\mu$, it is easily seen that $\Delta_{\mu}$ acts as a classical differential operator on the space $H_{\mu}$ and as a generalized one on $H_{\mu}^{* \prime}$, while that the conventional operator $\Delta_{\mu}^{*}$ acting on $H_{\mu}^{*}$ is understood as a distributional operator on $H_{\mu}^{\prime}$. These facts are justified in view of the inclusions $H_{\mu} \subset H_{\mu}^{*}$ and $H_{\mu}^{*} \subset H_{\mu}^{\prime}$, since $\Delta_{\mu}^{*}$ is the adjoint operator of $\Delta_{\mu}$.

Remark 6. Assume that $\mu$ is any real number. The generalized transformation $\mathcal{H}_{\mu}^{\prime}$ can be similarly defined on the space ${H^{\prime}}_{\mu}$ as the adjoint operator of $\mathcal{H}_{\mu}$ on $H_{\mu}$, namely,

$$
\left\langle\mathcal{H}_{\mu}^{\prime}{ }^{\prime} g, \Psi\right\rangle=\left\langle g, \mathscr{H}_{\mu} \Psi\right\rangle, g \in H^{\prime}{ }_{\mu}, \Psi \in H_{\mu}
$$

Finally, set $\psi=\mathcal{H}_{\mu} \Psi$. Then, because of Theorem 3.2-(a), (3.8) becomes

$$
\left\langle\mathcal{H}_{\mu}^{\prime} g, \mathscr{H}_{\mu} \Psi\right\rangle=\langle g, \psi\rangle, g \in H^{\prime}{ }_{\mu}, \psi \in H_{\mu}
$$

Again this expression can be considered as an exact transcription to generalized functions of the mixed Parseval relation (2.20). It is fulfilled that $\mathcal{H}_{\mu}^{\prime} g=\mathscr{H}_{\mu}^{\prime} g$ whatever be $g \in H_{\mu}^{*}$, as well.

An analogous result to Theorem 3.3 can be inferred from Theorem 3.2-(a):

Theorem 3.4. The generalized complementary Hankel transformation $\mathcal{H}_{\mu}^{\prime \prime}$, as given by (3.8), is an automorphism on $H^{\prime}{ }_{\mu}$ independently of the value of $\mu \in R$.

Next we collect some operation-transform formulas.

Theorem 3.5. Let $\mu$ be an arbitrary real number. For all $\phi \in H_{\mu}$,

$$
\begin{aligned}
\mathscr{H}_{\mu}\left\{\Delta_{\mu} \phi\right\} & =-y^{2} \mathcal{H}_{\mu} \phi \\
\Delta_{\mu} \mathcal{H}_{\mu} \phi & =\mathcal{H}_{\mu}\left(-x^{2} \phi\right)
\end{aligned}
$$

If $\psi \in H_{\mu}^{*}$, then

$$
\begin{aligned}
\mathscr{H}_{\mu}\left\{\Delta_{\mu}^{*} \psi\right\} & =-y^{2} \mathcal{H}_{\mu}^{*} \psi \\
\Delta_{\mu}^{*} \mathcal{H}_{\mu} \phi & =\mathscr{H}_{\mu}\left(-x^{2} \phi\right)
\end{aligned}
$$

For every $f \in H_{\mu}^{* \prime}$ one has

$$
\mathscr{H}_{\mu}\left\{\Delta_{\mu} f\right\}=-y^{2} \mathcal{H}_{\mu} f
$$

whereas

$$
\mathscr{H}_{\mu}^{\prime}\left\{\Lambda_{\mu}^{*} g\right\}=-y^{2} \mathscr{H}_{\mu}^{\prime} g
$$

holds for any $g \in H^{\prime}{ }_{\mu}$.

Remark 7. The Cauchy problem posed by W. Y. Lee [5]

$$
\begin{aligned}
& \frac{\partial u}{\partial t}=P\left(\Delta_{\mu, x}\right) u(x, t) \\
& u(x, 0)=f(x)
\end{aligned}
$$

where $f(x)$ is a known member of $H_{\mu}^{*}, P$ denotes a polynomial with constant coefficients and the unknown function $u(x, t)$ belongs to $H_{\mu}^{*}$, can now be solved by applying the transformation $\mathcal{H}_{\mu}$ not only for $\mu \geq-1 / 2$, but whatever for be the real value of the parameter $\mu$. This and above results justify that the transformation $\mathscr{H}_{\mu}$ be called the generalized Schwartz's Hankel transformation of arbitrary order. 


\section{REFERENCES}

[1] Altenburg, G.: "Bessel-Transformation in Räumen von Grundfunktionen über dem Intervall $\Omega=(0, \infty)$ und deren Dualräumen," Math. Nachr. 108 (1982), 197-218.

[2] Dube, L. S. and Pandey, J. N.: "On the Hankel Transform of Distributions," Tôhoku Math. J., 27 (1975), 337-354.

[3] Gray, A., Matthews, G. B., and MacRobert, T. M.: "A Treatise on Bessel Functions and Their Applications to Physics." MacMillan, London, 1952.

[4] Koh, E. L.: "The Hankel Transformation of Negative Order for Distributions of Rapid Growth," SIAM J. Math. Anal., 1 (1970), 322-327.

[5] Lee, W. Y.: "On Schwartz's Hankel Transformation of Certain Spaces of Distributions," SIAM J. Math. Anal., $\underline{6}$ (1975), 427-432.

[6] Macauley-Owen, P.: "Parseval's Theorem for Hankel Transform," Proc. London Math. Soc., 45 (1939), 458-474.

[7] Méndez Pérez, J. M. R.: "On the Bessel Transforms," Jñānābha, 17 (1987), 79-88.

[8] Méndez Pérez, J. M. R.: "On the Bessel Transformation of Arbitrary Order," Math. Nachr., 136 (1988), 233-239.

[9] Méndez Pérez, J. M. R.: "A Mixed Parseval Equation and the Generalized Hankel Transformation," Proc. Amer. Math. Soc., 102 (1988), 619-624.

[10] Shuitman, A.: "On a Certain Test Function Space for Schwartz's Hankel Transform," Delft Prog, Rep., 2 (1977), 192-206.

[11] Schwartz, L.: "Théorie des Distributions," Herman, Paris, 1966.

[12] Schwartz, A. L.: An Inversion Theorem for Hankel Transform," Proc. Amer. Math. Soc., 22 (1969), 713-717.

[13] Titchmarsh, E. C.: "Introduction to the Theory of Fourier Integrals," Oxford Univ. Press, London, 1959.

[14] Watson, G. N.: A Treatise on the Theory of Bessel Functions." Cambridge Univ. Press, Cambridge, 1958.

[15] Zemanian, A. H.: "A Distributional Hankel Transformation," SIAM J. Appl. Math., 14 (1966), 561-576.

[16] Zemanian, A. H.: "Hankel Transforms of Arbitrary Order," Duke Math. J., 34 (1967), 761-769.

[17] Zemanian, A. H.: "Generalized Integral Transformations," Interscience, N.Y. 1968. 


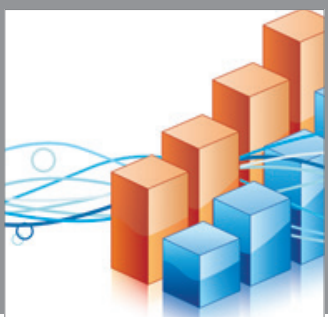

Advances in

Operations Research

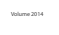

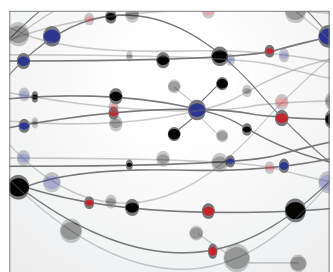

\section{The Scientific} World Journal
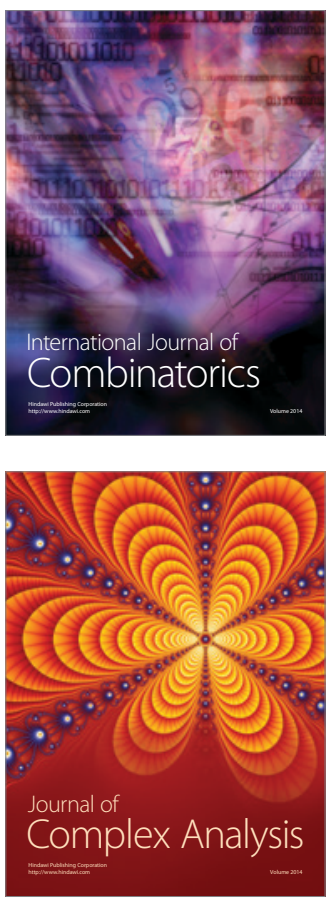

International Journal of

Mathematics and

Mathematical

Sciences
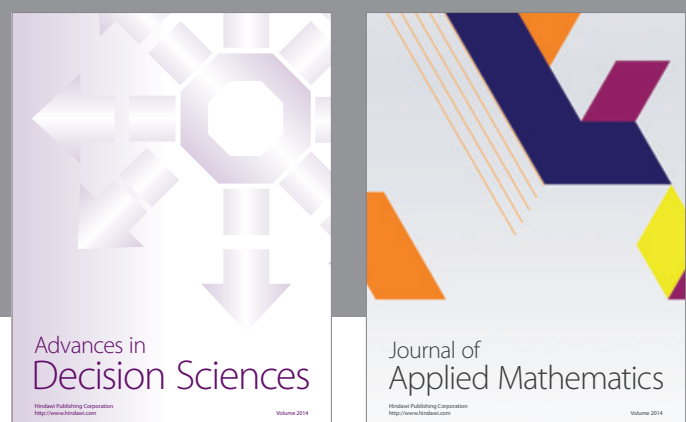

Journal of

Applied Mathematics
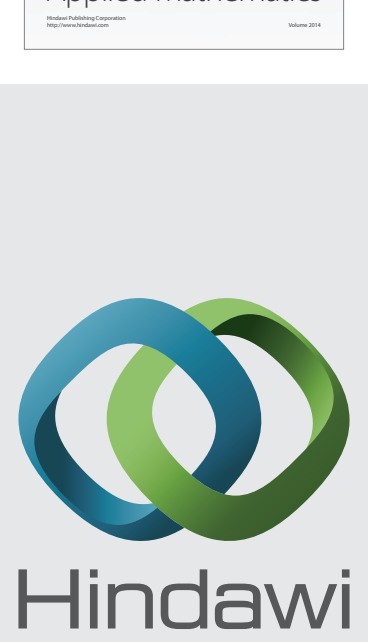

Submit your manuscripts at http://www.hindawi.com
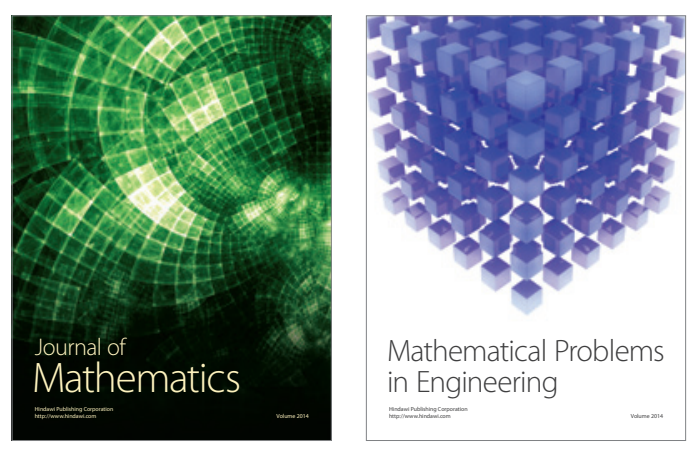

Mathematical Problems in Engineering
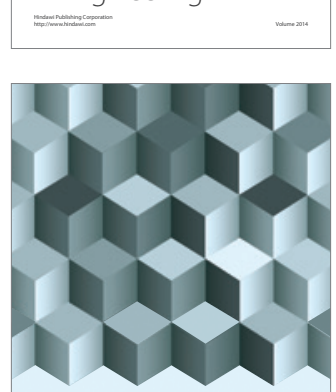

Journal of

Function Spaces
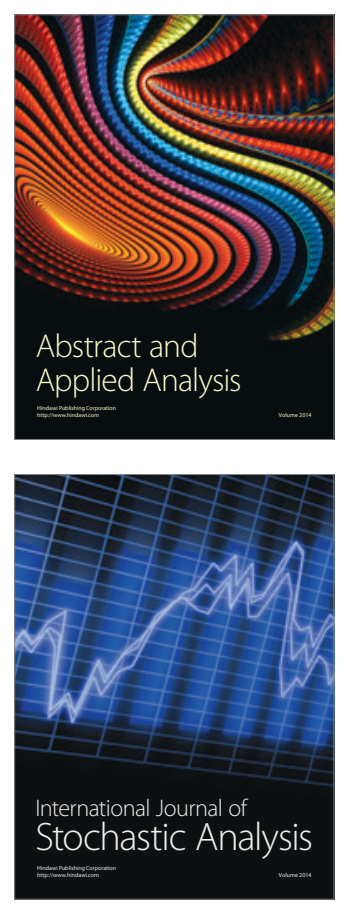

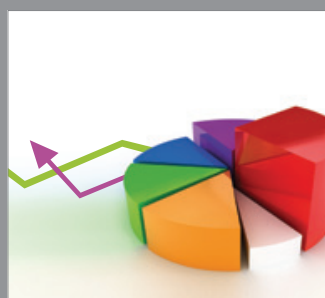

ournal of

Probability and Statistics

Promensencen
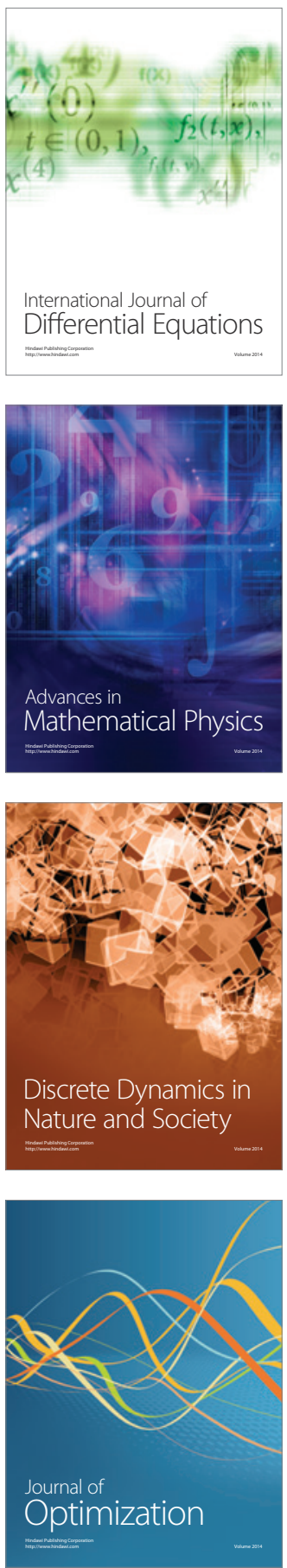